University of Nebraska - Lincoln

DigitalCommons@University of Nebraska - Lincoln

Comparison of dynamic compression behavior of single crystal sapphire to polycrystalline alumina

\author{
G. J. Kleiser \\ Air Force Research Laboratory, Eglin AFB, FL \\ L.C. Chhabildas \\ Air Force Research Laboratory, Eglin AFB, FL \\ W.D. Reinhart \\ Sandia National Laboratories, Albuquerque, NM
}

Follow this and additional works at: https://digitalcommons.unl.edu/usdoepub

Part of the Bioresource and Agricultural Engineering Commons

Kleiser, G. J.; Chhabildas, L.C.; and Reinhart, W.D., "Comparison of dynamic compression behavior of single crystal sapphire to polycrystalline alumina" (2011). US Department of Energy Publications. 117. https://digitalcommons.unl.edu/usdoepub/117

This Article is brought to you for free and open access by the U.S. Department of Energy at DigitalCommons@University of Nebraska - Lincoln. It has been accepted for inclusion in US Department of Energy Publications by an authorized administrator of DigitalCommons@University of Nebraska - Lincoln. 


\title{
Comparison of dynamic compression behavior of single crystal sapphire to polycrystalline alumina
}

\author{
G.J. Kleiser ${ }^{\mathrm{a}, *}$, L.C. Chhabildas ${ }^{\mathrm{a}}$, W.D. Reinhart ${ }^{\mathrm{b}}$ \\ ${ }^{a}$ Air Force Research Laboratory, 101W Eglin Blvd, Eglin AFB, FL 32578, USA \\ ${ }^{\mathrm{b}}$ Sandia National Laboratories, PO Box 5800, Albuquerque, NM 87185, USA
}

\section{A R T I C L E I N F O}

Article history:

Available online 30 December 2010

\section{Keywords:}

Shock loading

Single crystals

Alumina

Sapphire

Dynamic strength

\begin{abstract}
A B S T R A C T
Due to the considerable interest in the shock loading behavior of aluminum oxide whether it is in the polycrystalline phase or in the single crystal phase well-controlled experiments were conducted to probe differences in shock loading behavior between these two materials. Previous studies concluded that the behavior was similar but careful examination of well-controlled experiments has revealed the two materials are different.Although the experimental results appear to have the same behavior in the shock velocity vs. particle velocity plane, they are considerably different in the stress-volume compression plane and evidence is provided that indicates the single crystal remains crystalline up to the stresses imposed for this analysis. This is an extremely interesting observation since it has many implications including developing dynamic material models capable of transitioning between individual grains and polycrystalline material.
\end{abstract}

(c) 2010 Published by Elsevier Ltd.

\section{Introduction}

Due to the strength to weight properties of alumina and its individual constituent, sapphire, there has been considerable interest in the dynamic compression behavior of these materials particularly the single crystal properties of sapphire and how they affect polycrystalline alumina. Sapphire is commonly described by a hexagonal unit cell containing six formula units [1]. The basal planes are separated by $12.991 \AA$, and the axis separating the basal planes is defined as the c-axis, while the lattice parameter in the basal plane itself is determined to be $4.785 \AA$ [2]. It is not uncommon in the literature to refer to the c-axis sapphire as $z$-cut sapphire. In this paper, alumina is referred to as the polycrystalline material, specifically Coors AD 995, and is a sintered (powdered) mixture of all possible single crystal orientations. Early studies for sapphire have indicated the dependence of the Hugoniot Elastic Limit (HEL) to its specific orientation $[3,4]$ yet, there is considerable variation in its HEL even for the same orientation. The intent to use ceramics as an armor material has spurned additional investigations both for sapphire and alumina in an effort to improve the ability to model these materials [5-14]. These studies confirm the large variations in HEL for sapphire. Time-resolved particle velocity profile (VISAR) measurements of the elastic precursor obtained

\footnotetext{
* Corresponding author.

E-mail address: geremy.kleiser@eglin.af.mil (G.J. Kleiser).
}

from shocked sapphire profiles above the HEL are suggestive of elastic precursor decay which is followed by oscillatory variations in particle velocity. This is generally not repeatable and both the HEL and the fluctuations in particle velocity are unique to each experiment since the phenomenon is due to heterogeneous yielding that is dependent both on the nucleation time for slip planes and also the peak loading stress. The values for HEL for sapphire vary from 12 to $22 \mathrm{GPa}$ and are considerably higher than those indicated for polycrystalline alumina which is measured to be approximately $7 \mathrm{GPa}$. This is not surprising since it is well known that single crystals exhibit large dynamic yield strengths when compared to their polycrystalline form. In addition to the HEL variation, there has been experimental evidence both for alumina $[15,16]$ and sapphire [4] that the material loses partial or total strength when compressed to high shock pressures. Mashimo [9], based on shock compression measurements of a-axis single crystals up to over $100 \mathrm{GPa}$, suggested the existence of a phase transformation. This was not confirmed by particle velocity VISAR measurements of shock compression behavior on both a-axis and c-axis sapphire up to $100 \mathrm{GPa}$ [10-12]. When all the past measurements of shock compression data for sapphire and alumina is combined as an integrated plot in the shock velocity- particle velocity plane, considerable scatter is observed as indicated in Fig. 1. The scatter results from the variety of different experimental techniques and the inherent error associated with techniques such as flash gaps, pin contactors, and inclined mirror methods. For example at a particle velocity of $2 \mathrm{~km} / \mathrm{s}$ the difference between 


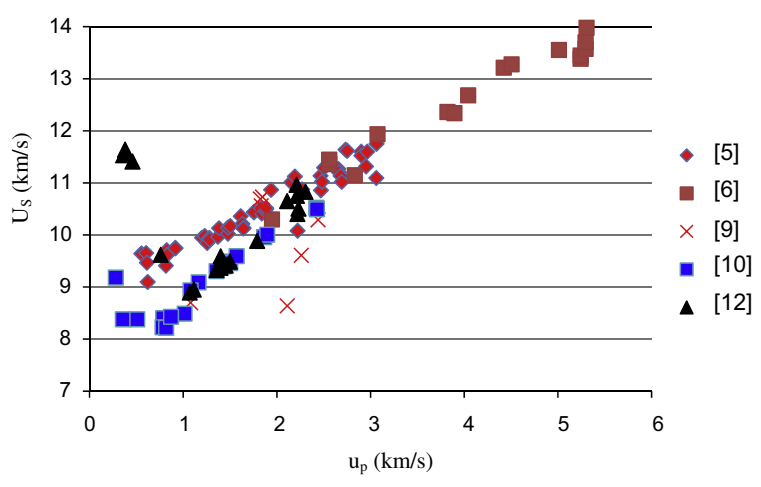

Fig. 1. Data variation for alumina and sapphire due to experimental technique. See references [5-12].

maximum and minimum shock velocity is around $7 \%$, while at around $1 \mathrm{~km} / \mathrm{s}$ the variation is even more. One reason for the large variation near $1 \mathrm{~km} / \mathrm{s}$ is due to the proximity of the HEL and the heterogeneous yielding process. Other reasons include the sensitivity of experimental techniques to different test methods. For example, how sensitive is the technique to the elastic-plastic twowave structure? This is especially true for materials that have a high value for the elastic precursor wave. A seven percent uncertainty in shock velocity particle velocity measurements would translate into an uncertainty of around $14 \%$ in the pressure-volume plane. This scatter in data has led to the obvious conclusion that the high pressure compression behavior for sapphire and alumina are the same. It is the purpose of this paper to compare the published results of previous studies on alumina and sapphire that were conducted using the same method and VISAR velocity interferometric techniques [10-12]. Even though the results of these experiments have been published separately it is beneficial to compare them in a single paper to highlight the differences and accentuate the similarities so that one does not use or refer to the data interchangeably. In particular, it is noted that the high pressure compression curve i.e. the Hugoniot for shocked sapphire and alumina are not the same and is not due to the differences in the HEL's for the two materials, because for both materials the shock compression pressure-volume curve and the mean pressure-volume curve are the same within the experimental uncertainty. For completeness the following sections briefly describe the experimental techniques and the analysis used to obtain not only the shock Hugoniot but also the mean pressure curve. It is conjectured that these differences are primarily due to sapphire remaining a single crystal even at such high compression stresses.

\section{Materials \& experimental method}

Materials for this study were high quality sapphire with [0001] orientation having an average density of $3.989 \mathrm{~g} / \mathrm{cm} 3$. The samples were cut from boules of synthetically grown sapphire such that the direction of shock propagation was along the $\mathrm{C}$ - axis of the material. The longitudinal elastic wave speed is $11.19 \mathrm{~km} / \mathrm{s}$. Based on non linear elastic behavior the elastic wave speed in the elastic regime varies as $\mathrm{Ce}=11.19+1.0$ up to a particle velocity of $0.6 \mathrm{~km} / \mathrm{s}$. The aluminum oxide $\left(\mathrm{Al}_{2} \mathrm{O}_{3}\right)$ used in this study is referred to as Coors AD995 alumina. Its composition consists of 99.5\% alumina and the remainder of the material is aluminosilicate glass. The density of the material $\left(\mathrm{Al}_{2} \mathrm{O}_{3}\right)$ was $3.89 \mathrm{~g} / \mathrm{cm}^{3}$ and the average longitudinal and shear wave speed was $10.56 \mathrm{~km} / \mathrm{s}$ and $6.24 \mathrm{~km} / \mathrm{s}$ respectively. This yields an estimate of $7.71 \mathrm{~km} / \mathrm{s}$, 0.234 and 231.7 GPa for the bulk wave velocity, Poisson's ratio and the bulk modulus, respectively.
Compressive shock, reshock and release, and cyclic waves were produced in polycrystalline alumina and $z$-cut sapphire using a two-stage light gas gun. The experimental configuration used for this study on the two-stage light gas gun is shown in Fig. 2. The gas gun utilized a $28 \mathrm{~mm}$ bore diameter with projectile velocities approaching $8 \mathrm{~km} / \mathrm{s}$ and incorporated a projectile velocity measuring system called the Optical Beam Reflector (OBR) [17] that accurately measured projectile velocity to better than $0.2 \%$. Electrical self-shorting pins were also used for impact planarity (nominally better than 6 milliradians) and diagnostic triggering. As shown in Fig. 2, the projectile was faced with either the ceramic Coors AD995 or Z-cut sapphire and was backed with a plastic (TPX) of low shock impedance for release experiments, or a high shock impedance material (tantalum or copper), for reloading experiments. The target configuration in Fig. 2 used a symmetric impact configuration meaning that both the projectile material and the target material were the same in each experiment. The target disk similar to that mounted on the projectile had a single crystal lithium-fluoride disk bonded to the back of the target sample. The lithium-fluoride was an optical quality disk, lapped and polished and was typically flat to within a few bands of sodium light. One surface of the lithium-fluoride was diffused and approximately $100 \mathrm{~nm}$ of aluminum was vapor deposited on the lapped surface before being glued to the target. The particle velocity histories resulting from impact were measured at the target/lithium-fluoride window [18] interface using a velocity interferometer, VISAR [19]. The Doppler shifted interference fringes measured with the VISAR were converted to a time-resolved velocity history with the amplitude resolution being approximately $2 \%$ per fringe and typically two to three fringes were achieved in the interface acceleration resulting from the compressive shock front, indicating that the peak particle velocity measurements were accurate to better than $1 \%$.

\section{Experimental results}

\subsection{Elastic E plastic-waves and off-Hugoniot states}

The measured wave profiles were used to determine the peak longitudinal stress states achieved in the initial shock compression process which is referred to as the Hugoniot properties. The Hugoniot elastic limit stress, $\left(\sigma_{\text {hel }}\right)$, was determined using the relation:

$\sigma_{\text {hel }}=\left(\rho_{0} C_{1} u_{e}\right)$

where $\rho_{\mathrm{o}}$ is the initial density of the ceramic, $C_{1}$ the elastic longitudinal wave speed, and $u_{\mathrm{e}}$ is the in-material particle velocity measurement prior to transition to a plastic-wave. The in-material particle velocity was determined through the impedance matching relation where the $w$ subscript indicates the window material and subscript $m$ represents the material of interest

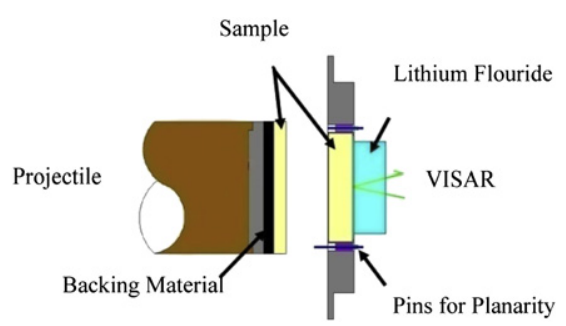

Fig. 2. Experimental impact configuration used for shock loading, release/reshock experimental results described in this paper. 
$u_{e}=u_{w} \frac{\left(Z_{w}+Z_{m}\right)}{2 Z_{m}}$

The planar impact produced a compressive wave of uniaxial strain, which propagated across the target specimen and into the lithium-fluoride window. The measured velocity exhibited a two-wave structure. The subsequent structure following the elastic precursor represented pressure hardening of the material and the two-wave structure was the result of a transition from elastic to plastic deformation. As compression within the shock increased during the shock loading process, shear stresses exceeded the critical strength of the material (HEL) and plastic deformation occurred in the observed second wave. Because finite rise times were measured for the plastic-wave, the plastic-wave velocity, $U_{\mathrm{sp}}$, was taken at the center of the wave. All the experiments were performed using symmetric impact technique, therefore the particle (material) velocity, $u_{\mathrm{ph}}$, behind the shock front was exactly one-half the impact velocity. The Hugoniot stress, $\sigma_{\mathrm{ph}}$, and strain, $\epsilon_{\mathrm{ph}}$, behind the plastic-wave front were estimated using the following relations:

$\sigma_{\mathrm{ph}}=\sigma_{e}+\rho_{0} U_{\mathrm{sp}}\left(u_{\mathrm{ph}}-u_{\mathrm{e}}\right)$

$\epsilon_{\mathrm{ph}}=\epsilon_{e}+\frac{\left(u_{\mathrm{ph}}-u_{e}\right)}{U_{\mathrm{sp}}}$

Thus, these time-resolved experiments yielded a wealth of data (the initial elastic wave speed, shock velocity, particle velocity, stress, strain, and the Hugoniot elastic limit) merely from a detailed analysis of the loading profile.

All the experiments conducted in this investigation, also evaluated the off-Hugoniot states of Coors AD995 alumina and z-cut sapphire as it was allowed to release and reload from its initial compression. An incremental form of the conservation equations given by the relations:

$\sigma=\sum \rho_{0} c \Delta u$

$\epsilon=\sum \Delta u / c$

were used to estimate the final released or reshocked stress, $\sigma$ and strain, $\epsilon$, respectively. The Lagrangian-wave velocity, $c$, corresponds to the current material particle velocity corresponding to the change, $\Sigma \Delta u$. Details of this analysis method is given elsewhere [7], and will not be repeated here.

\subsection{Hugoniot elastic limits}

The Hugoniot elastic limit of Coors AD995 varied from about 7.0 GPa to 7.9 GPa [11]. This appears slightly higher than previous studies performed at lower pressure states. An explanation for this could be inferred from a previous study in crystalline quartz [20], sapphire [4], and beryllium [21] that has indicated different values of HEL for different crystal orientation. This is because different slip systems are activated during the dynamic yielding process. In a polycrystalline material, all these different slip systems are randomly distributed and hence the results will be dominated by the weaker slip systems that will yield at a lower stress. This will result in an initial elastic shock followed by a ramp - indicative of a work hardening type behavior. As the Hugoniot stress is increased, as in this study, HEL in fact increases slightly over the previous studies of alumina [22,10]. The Hugoniot elastic limit in alumina will be overdriven when the shock velocity measurement exceeds the elastic wave velocity of $10.74 \mathrm{~km} / \mathrm{s}$.

Another interesting feature observed in this study is the transition from initial yielding at the HEL to the Hugoniot state. The post
HEL yielding process in polycrystalline alumina up to $60 \mathrm{GPa}$, is considerably ramped suggesting a work hardening type of process or heterogeneous yielding from different slip systems. The ramp region is followed by a plastic-wave that has a finite rise time. As the pressure is increased to $100 \mathrm{GPa}$, the plastic-wave approaches the elastic wave velocity, and the ramping has all but disappeared. It should also be noted however, that even at $100 \mathrm{GPa}$, the elastic limit is not yet overdriven.

The measured elastic wave profiles were used to determine the Hugoniot Elastic Limit of z-cut sapphire. Sapphire is known to behave as an elastic material below its Hugoniot elastic limit [3]. Therefore the shock velocity/particle velocity in the linear elastic regime given by Eq. (7) is used to determine the elastic shock velocity, where $U_{\text {se }}$ and $u_{e}$ is the elastic shock and particle velocity of sapphire respectively.

$U_{\text {se }}=11.19+1.0 u_{e}$

In this study, the average Hugoniot elastic limit for sapphire is determined to be 22 GPa It is close to a factor of three higher than what has been reported for polycrystalline alumina (7 GPa) [11], and is not unusual for single crystals to have much higher strengths than polycrystalline materials.

\subsection{Shock velocity vs. particle velocity}

In this study, symmetric impact conditions were utilized. The particle velocity estimates were one-half the impact velocity, and measured to better than $0.2 \%$, while the shock velocity estimates were better than $1 \%$. The shock velocity $v$ s. particle velocity fit given by Eqs. (7) and (2) were fit over the particle velocity range of $0.5 \mathrm{~km} / \mathrm{s}$ to $2.5 \mathrm{~km} / \mathrm{s}$. It should be noted that the elastic precursor is still not overdriven at symmetric impact velocities of $4.86 \mathrm{~km} / \mathrm{s}$, i.e. at a particle velocity of $2.43 \mathrm{~km} / \mathrm{s}$.

The variation in shock velocity vs. particle velocity for alumina is shown in Fig. 3. The shock velocity measurements below a particle velocity measurement of $0.5 \mathrm{~km} / \mathrm{s}$ indicate a quasielastic behavior. For polycrystalline alumina, the dynamic yielding processappears to be incomplete below $20 \mathrm{GPa}\left(\sim 0.75 \mathrm{~km} / \mathrm{s} u_{p}\right.$ in Fig. 3). In contrast, the shock velocity measurements above a particle velocity of $0.5 \mathrm{~km} / \mathrm{s}$ suggest that the yielding process may be near completion. This implies the existence of distributed shear stress states for polycrystalline alumina and is consistent with the large yield strength measurements observed for single crystal sapphire. As indicated in Fig. 3, the observed shock velocity

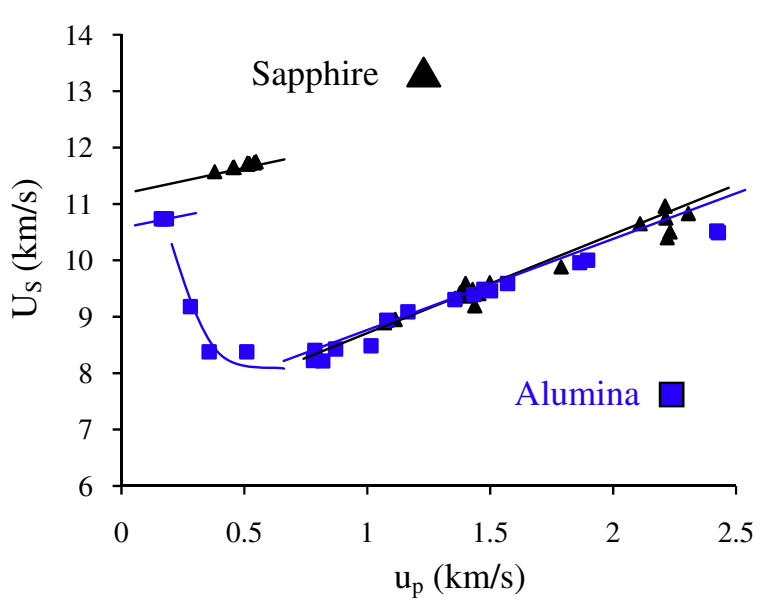

Fig. 3. Shock velocity vs. particle velocity variation for alumina and z-cut sapphire. 
vs. particle velocity up to $100 \mathrm{GPa}$ is plotted with a least squares fit to the data over the particle velocity range of $0.78-2.4 \mathrm{~km} / \mathrm{s}$ providing the relation:

$U_{s}(\mathrm{~km} / \mathrm{s})=1.299 u_{\mathrm{ph}}+7.455$

In the sapphire velocity history profiles, the loading profile had a two-wave structure and a distinct rise time to the compressed, shocked state. The shock velocity state was estimated from the arrival time [12]. The shock wave speed, $U_{S}$, in sapphire in the shocked state fits the relation:

$U_{s}=1.533 u_{\mathrm{ph}}+7.27$

where, $u_{\mathrm{ph}}$, is the particle velocity of the material. The shock velocity particle velocity relation given by Eq. (9) is shown in Fig. 3.

Since these experiments were conducted to a very high precision, we can observe the differences in the $U_{s}$ vs $u_{\mathrm{ph}}$ behavior for polycrystalline alumina and z-cut sapphire. To the first order one may assume that the behavior is the same. This suggests that the respective shock velocities for polycrystalline alumina and $z$-cut sapphire are parallel to each other. In particular, the differences in the Hugoniot stress vs. particle velocity behavior for the two materials will be given by the differences in the HEL. This does not, however, indicate that the stress-volume compression will be the same because the Hugoniot Elastic Limit (HEL), is different for the two materials. This is shown in Fig. 4 where the stress versus specific volume is plotted for both alumina and z-cut sapphire.

\subsection{Stress vs. strain}

The stress vs. specific volume compression behavior is shown both for alumina and $z$-cut sapphire in Fig. 4 . As indicated in the graph the elastic compression is indicated up to around $7.9 \mathrm{GPa}$ for alumina and around $22 \mathrm{GPa}$ for sapphire. It is a bit surprising to note that the elastic limit for polycrystalline alumina is substantially lower by almost a factor of 3 , but is understandable if you consider that the loading behavior for the alumina indicates considerable "work hardening" prior to plastic-wave arrival, while the loading profile for sapphire does not indicate a similar loading structure. This is, therefore, further evidence that the yielding mechanisms for a single crystal versus polycrystalline alumina are totally different.

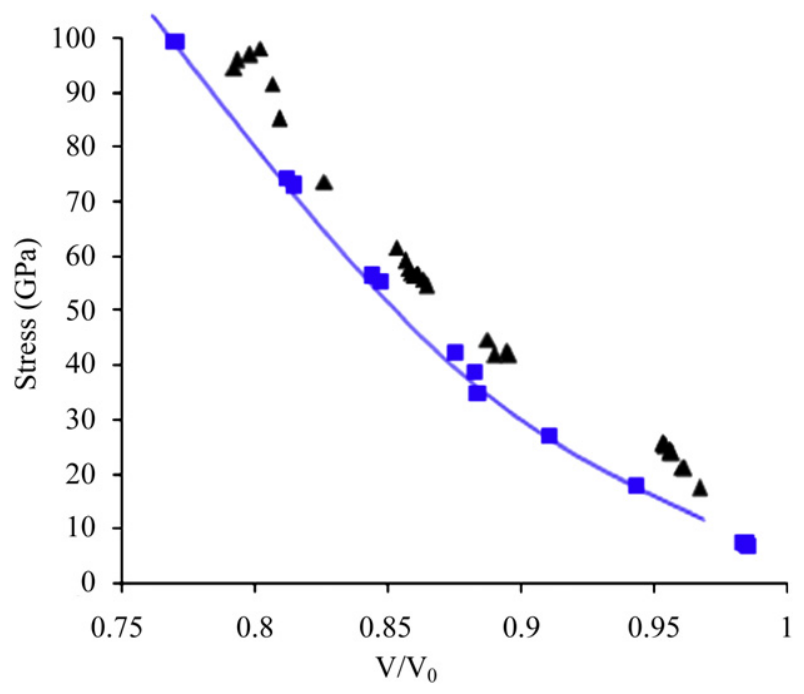

Fig. 4. Stress-volume compression behavior of alumina and z-cut sapphire.
As shown in Fig. 4, the end states, mainly, the shock Hugoniot for these two different materials are not the same. There have been many investigations conducted and reported in the literature for alumina, corundum, and sapphire in the past and when all the data are plotted together little distinction can be made. The past experimental data is then concluded to have a large uncertainty which can be attributed to variation in-material purity, loading technique and diagnostic sensitivity. Therefore the experimental determinations are not sufficiently accurate in order to see the differences between the two materials. In these studies, we have reported only shock Hugoniot compression data acquired using smooth-bore gun technology [23], combined with high speed nanosecond time-resolution velocity interferometry [19]. The uncertainty in the measurements shown in Fig. 4 are less than $3 \%$ and would be of the order of $1.5 \mathrm{GPa}$ at around $50 \mathrm{GPa}$. The difference in stress at a given volume compression is of the order of $10 \mathrm{GPa}$ over the entire compression regime as indicated in Fig. 4. It is evident that these differences are due to differences in the elastic loading limit/behavior of alumina and z-cut sapphire. It is also indicating that the z-cut sapphire is stiffer than the polycrystalline alumina. We also believe that the $z$-cut sapphire is maintaining its crystalline structure as verified by elastic release and reshock wave speed measurements in sapphire at $56 \mathrm{GPa}$ (at a particle velocity of $1.4 \mathrm{~km} / \mathrm{s}$ ) [12] where the measured wave speed was within a $1 \%$ of the extrapolated wave speed given by Eq. (7).

\subsection{Strength in the shocked state}

The main reason for conducting companion reshock and release experiments are shown in the stress vs. volume plane. In all companion reshock and release experiments conducted on alumina [11] and $z$-cut sapphire [12], the leading edge of the release wave or the reshock wave, traverses at an elastic wave velocity thus providing evidence that the material is reloading or unloading elastically. This phenomena has been observed previously in 6061-T6 aluminum, [24], tungsten [25], beryllium [26], copper [27], and was observed in previous studies of Coors AD995 alumina even at its Hugoniot elastic limit [28]. Additionally, this experimental technique provides the ability to determine a shock hydrostat [24] at very high dynamic stresses, which is more accurate than extrapolating the hydrostatic data, because the dynamic hydrostat will be the mean value of the reshock and release end states arrived at from a common Hugoniot state.

In [24] the method used for determining strength is graphically depicted. In particular, the relation between the Hugoniot stress $\left(\sigma_{h}\right)$, mean pressure $(P)$, and shear stress $\left(\tau_{h}\right)$ is given by:

$\sigma_{h}=P+\frac{4}{3} \tau_{h}$

$\sigma_{\max }=P+\frac{4}{3} \tau_{c}$

$\sigma_{\min }=P-\frac{4}{3} \tau_{c}$

where $\tau_{h}$ is the shear stress of the material at the shocked or Hugoniot state, $\tau_{c}$ is the maximum shear stress state that the material can sustain, and $\mathrm{P}$ is the mean pressure of the material. $\sigma_{\max }$ and $\sigma_{\min }$ will be the stress states determined from the reshock and release experiments at the common Hugoniot strain $\epsilon_{h}$.

As indicated in Fig. 5, states $+\tau_{c}$ and $-\tau_{c}$ are defined as maximum or minimum shear stress states because the Lagrangianwave velocity transitions from elastic wave to the bulk wave at those states. Combining Eqs. (10-12), the following expressions are obtained: 


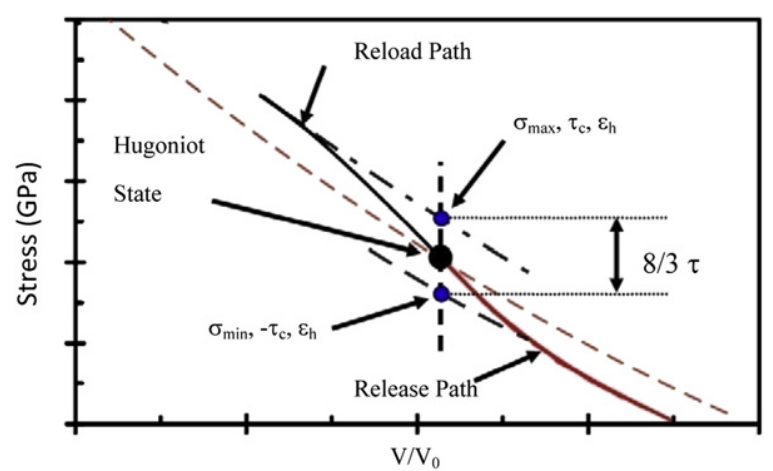

Fig. 5. Reshock/release experimental results used to depict technique to estimate the shear stress state, $\tau_{h}$, and the maximum strength, $\tau_{c}$, the material can sustain at a Hugoniot strain of $\epsilon_{h}$. This allows us to determine the mean stress state, $P$, for the materials.

$\tau_{c}+\tau_{h}=\frac{3}{4}\left(\sigma_{h}-\sigma_{\min }\right)$

$\tau_{c}-\tau_{h}=\frac{3}{4}\left(\sigma_{\max }-\sigma_{h}\right)$

$Y_{c}=2 \tau_{c}=\frac{3}{4}\left(\sigma_{\max }-\sigma_{\min }\right)$

whereby the critical strength $\left(Y_{c}\right.$, the maximum shear strength) is defined by the expression, $\tau_{h}$ very close to zero implies that the mean pressure state P, according to Eq. (10) reduces to $\sigma_{h}=P$, i.e., the Hugoniot states and the mean pressure states are identical. For an elastic-perfectly plastic material, $\tau_{c}=\tau_{h}$, giving $2 \tau_{c}$ the dynamic yield strength of the material and there would be an absence of elastic recompression. $\tau_{h}$ or $\tau_{c}$ increasing or decreasing with $\sigma_{h}$ would imply pressure dependent yield behavior, respectively.

The measured reshock and release wave velocity profiles, examples of which are shown in Fig. 5 are used to estimate the continuous reloading or release states from the shocked Hugoniot states. An incremental form of the conservation equations described by Eqs. (5) and (6) are used to estimate these offHugoniot states. The stress difference upon reshock is intentionally kept relatively small so that the elastic recompression upon reshock is not over driven.

As indicated in Fig. 5, both the reloading and release paths lie above and below the Hugoniot states, respectively, in all experiments -(all experiments are not shown) a phenomenon that is easily attributed to the strength in the shocked state. Using the prescription described above these measurements have been used to determine $\tau_{h}$, the shear stress at the shocked or Hugoniot state of the material, $\tau_{c}$ the maximum shear stress state that the material can sustain, and $P$ the mean pressure in the shocked state. Fig. 6 describes the variation of the average shear stress $\tau_{h}$, in the shocked state and the critical strength, $Y_{c}$ for alumina, and $z$-cut sapphire as determined from the unloading and reloading data $[11,12]$.

The variation of the derived quantities, $2 \tau_{c}$ the maximum strength alumina can sustain, and $\tau_{h}$ the shear stress of alumina in the shocked state as a function of shock stress is also shown in Fig. 6. $\tau_{h}$ is approximately zero both for alumina and $z$-cut sapphire. $2 \tau_{c}$ the yield strength of the material is increasing with increasing shock stress for alumina while it appears to be decreasing for sapphire. $\tau_{h}$ is approximately zero which implies that the shocked state for alumina and z-cut sapphire is equal to their mean pressure i.e., very close to the hydrostat. This is shown in Fig. 7 where the Hugoniot stress measurements are plotted as a function of strain over the stress range of 0-120 GPa for alumina and z-cut sapphire.

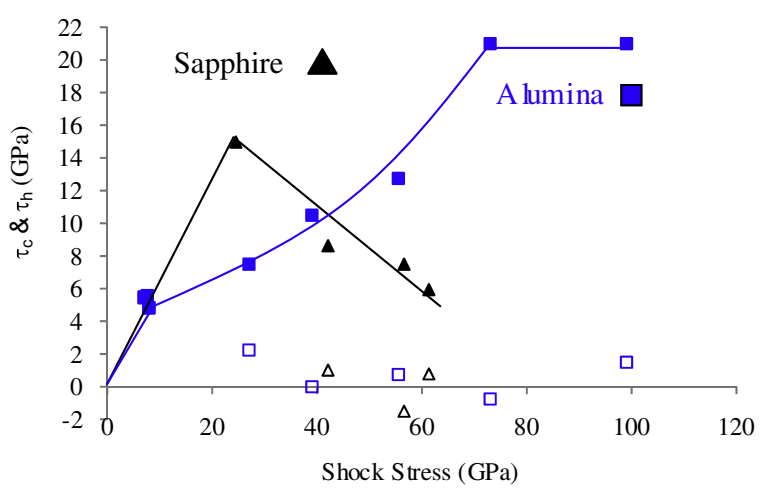

Fig. 6. Strength of alumina and $z$-cut sapphire in the shocked state.

Since the hugoniot shear stress is essentially zero the hugoniot stress and mean pressure or dynamic hydrostat are essentially the same. These measurements demonstrate the usefulness of using reshock and release experiments to determine not only the strength of the materials, but also the dynamic hydrostat even at megabar stresses. The two main conclusions that can be derived from these measurements is that the yield strength of alumina does increase with increasing stress - suggesting a pressure dependent yield, while the shears stress state of alumina suggest a collapse toward the hydrostat. It is not clear what the physical mechanisms are that is responsible for this behavior in alumina. For $z$-cut sapphire, however, even though the material collapses to the hydrostat (not unlike alumina), the yield strength $2 \tau_{c}$ does appear to be decreasing. Perhaps the most surprising result appears to suggest that the mean pressure curves for alumina and z-cut sapphire obtained from these reshock/release experiments are different, even though both materials collapse to their respective hydrostat on the Hugoniot. As indicated earlier, the mean pressure curve for alumina is resulting from an average which is a superposition of mean states of anisotropic grains of all possible orientations. In the case of z-cut sapphire the mean pressure curve is a result of only one orientation mainly the c-axis. Also, there is strong evidence that the material still preserves it single crystal shape. In this study, the shock propagates along the $z$-axis. How important does anisotropy play in the complex compression behavior of sapphire? What will be the shock compression behavior of sapphire when the shock propagates orthogonal to the $z$-axis namely along the a-axis? Should we anticipate the compression behavior of the a-axis single crystal to lie below the compression behavior of alumina? There is a strong interest in using the elastic

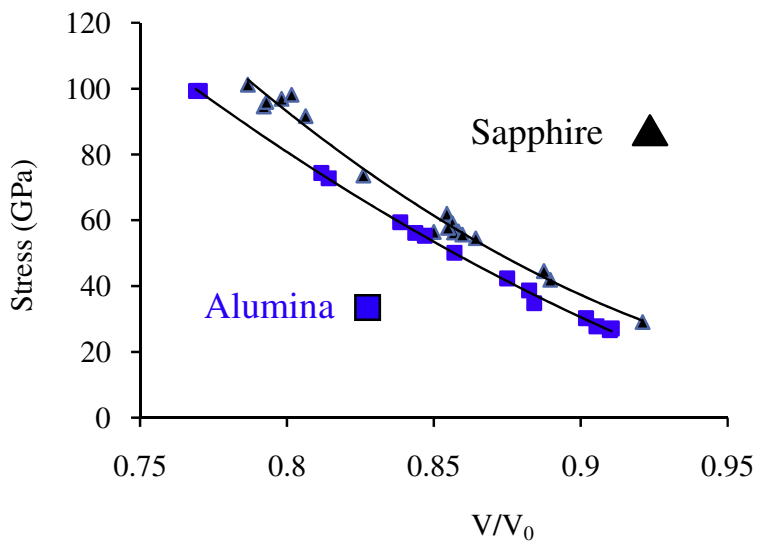

Fig. 7. Comparison of mean pressure with their respective Hugoniots for alumina and $z$-cut sapphire. 


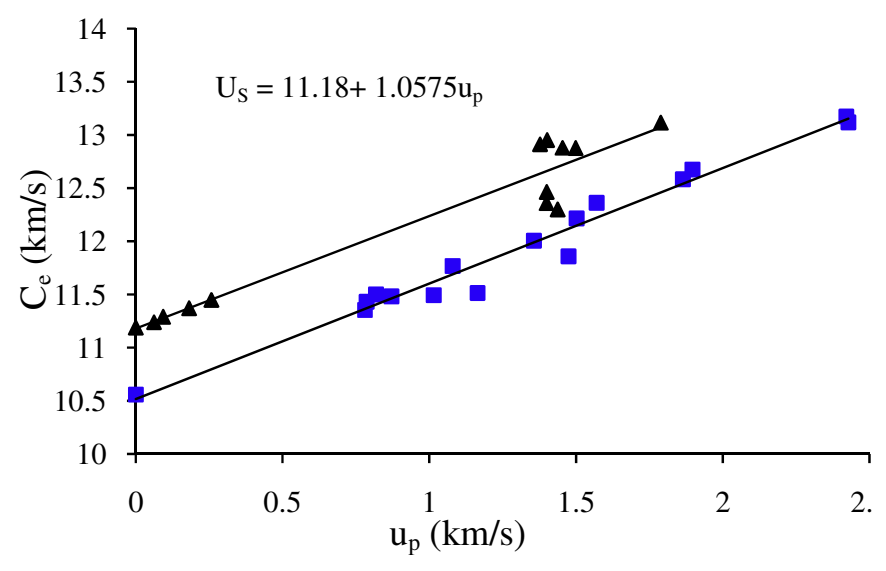

Fig. 8. Elastic release wave speed in the shocked state.

constants and compression behavior of single crystals as building blocks to developing equation of state of polycrystalline materials. This would indeed make an interesting study.

\subsection{Elastic wave speeds in the shocked state}

As further proof that the sapphire is remaining a single crystal during deformation the elastic wave velocities of the release waves are plotted in Fig. 8, and they show a distinct difference between polycrystalline alumina and the single crystal sapphire $[3,12]$ and alumina [11]. The low pressure data for sapphire up to a particle velocity of $0.25 \mathrm{~km} / \mathrm{s}$ is given by Barker and Hollenbach [3]. Since these low pressure experiments are conducted at and below the elastic limit the loading and release wave velocities are expected to be the same. As indicated in the figure there is a systematic difference in the elastic release wave velocities in the shocked state for sapphire and alumina up to a particle velocity of $2 \mathrm{~km} / \mathrm{s}$. A linear least squares fit to the data set for sapphire suggest that it can be represented by the equation $C_{e}(\mathrm{~km} / \mathrm{s})=11.18+1.0575 u_{p}$ and within the experimental uncertainty is very nearly identical to the liner shock velocity vs particle velocity relation for sapphire namely $U_{s}(\mathrm{~km} / \mathrm{s})=11.19+1.00 u_{p}$ [3]. This is the most convincing evidence that sapphire remains elastic and does not lose it single crystalline structure at very high shock compression pressures up to a particle velocity of $2 \mathrm{~km} / \mathrm{s}$. Additional release wave experiments are necessary to determine the nature of its crystalline structure with high precision above a particle velocity of $2 \mathrm{~km} / \mathrm{s}$.

\section{Summary}

The main emphasis of this paper is to carefully compare the results of shock compression data on shocked polycrystalline alumina and single crystal c-axis or z-cut sapphire. There is considerable scatter in the experimental data that has been published in the literature since the late sixties and early seventies on shock compressed alumina and single crystal sapphire. Hence a systematic comparison is not feasible. Only the results of wellcontrolled reshock and release experiments sing VISAR velocity interferometric techniques conducted on alumina and $z$-cut sapphire $[11,12]$ are used for comparison. These experiments were conducted to determine the strength of the materials and their respective dynamic hydrostat when these materials were shocked to stresses over 1 Mbar. The experiments on z-cut sapphire were conducted to evaluate the existence of phase transitions reported in the literature [9]. No unambiguous phase transformation in sapphire was detected. To summarize,
1) The stress-volume behavior of single crystal z-cut sapphire and polycrystalline alumina is significantly different beyond the experimental uncertainty, i.e., the shock Hugoniot when expressed in the stress-volume planes are different (Fig. 4).

2) Both materials collapse respectively to their shock hydrostats. In other words, within the experimental uncertainty. The shock Hugoniot for each material can also be represented as their mean pressure curves. (Fig. 7)

3) The leading edge of the elastic release wave velocities in shock compressed alumina and $\mathrm{z}$-cut sapphire are different as a function of particle velocity in the shocked state. (Fig. 3)

4) The leading edge of the elastic release wave velocity can be represented by an extrapolation of Barker's experimental data on z-cut sapphire at low stresses. This is strong evidence that the z-cut sapphire remains a single crystal even at high shock compressed stresses. (Fig. 8)

There are technical implications if $z$-cut sapphire remains a single crystal at extreme shock compressions up to and over a Mbar. If true, this would make a good experimental technique to determine the crystalline nature of the shocked single crystal. Needless to say, this needs to be verified using dynamic X-ray diffraction techniques $[29,30]$ to further complement these studies. $\mathrm{X}$-ray techniques, are limited to measurements of lattice dimensions at high stresses. If it is confirmed, then these test methodologies can be used to further correlate dynamic material properties such as strength and wave speeds in the compressed sate to its single crystalline structure. There is considerable interest in determining average dynamic properties of polycrystalline materials based on the elastic constants and their dependence at high stresses. For anisotropic crystals, such as sapphire, one would need to determine the single crystal properties of other orientations as well. The first step is to extend this study to other orientations such as a-axis crystals and other orientations. This would be the first step necessary to evaluate the concept of bridging different length scales.

\section{References}

[1] Alexander CS, Reinhart WC, Thornhill TF, Chhabildas LC. Armor options: a comparison of the dynamic response of materials in the aluminum oxidealuminum nitride family. Int J Applied Ceramics Technology 2010;7 (5):587-94.

[2] Bialas H, Stolz HJ. Lattice dynamics of sapphire (corundum). I. Phonon dispersion by inelastic neutron scattering. Z Phys B Condens Matter Quanta 1975;21(4):319-24

[3] Barker LM, Hollenbach RE. Shock-wave studies of PMMA, fused silica, and sapphire. J Appl Phys 1970;41:4208-26.

[4] Graham RA, Brooks WP. Shock-wave compression of sapphire from 15 to 420 kbars. The effects of large anisotropic compressions. Phys Chem Solids 1971;32:2311-30.

[5] Marsh SP. LASL shock hugoniot data. Berkely: University of California Press; 1980.

[6] Erskine D. High pressure hugoniot of sapphire: shock wave of condensed matter. New York: AIP Press; 1993.

[7] Grady DE. Shock wave compression of brittle solids. Mechanics Mater 1998;29:181-203.

[8] Mashimo T, Tsumoto K, Nakamura K, Noguchi Y, Fukuoka K, Syono Y. Highpressure phase transformation of corundum $\left(\mathrm{Al}_{2} \mathrm{O}_{3}\right)$ observed under shock compression. Geophys Res Lett 2000;27:2021-4.

[9] Mashimo T, Hanaoka Y, Nagayama K. Elastoplastic properties under shock compression of $\mathrm{Al}_{2} \mathrm{O}_{3}$ single crystal and polycrystal. J Appl Phys 1988;63: 327-36.

[10] Reinhart WD, Chhabildas LC, Mashimo T. Shock compression and release properties of Coors AD995 alumina in ceramic armor, materials by design, vol. 134. Maui, HI: The American Ceramics Society; 2002. 233-247.

[11] Reinhart WD, Chhabildas LC. Strength properties of Coors AD995 alumina in the shocked state. Int J Impact Eng 2003;29:601-19.

[12] Reinhart WD, Chhabildas LC, Vogler TJ. Investigating phase transitions and strength in single crystal sapphire using shock-reshock loading techniques. Int J Imp Engng 2005;33(1-12):655-69.

[13] Dandekar DP, Vaughan BAM, Proud WG. Shear strength of aluminum oxynitride. Shock Compression of Condensed Matter 2007;955:505-8. 
[14] Kanel GI, Nellis WJ, Savinykh AS, Razorenov SV, Rajendran AM. Response of seven crystallographic orientations of sapphire crystals to shock stresses of 16-86GPa. J App Phys 2009;109.

[15] Munson DE, Lawrence RJ. Dynamic deformation of polycrystalline alumina. J Appl Phys 1980;50:6272-82.

[16] Ahrens TJ, Gust WH, Royce EB. Material strength effect in the shock compression of alumina. J Appl Phys 1968;39:4610-6.

[17] Thornhill T, Reinhart WD, Konrad CH, Chhabildas LC. Accurate velocity measurements of the two-stage light-gas gun projectile, In: 51st Aeroballistic Range Assoc., 2000.

[18] Wise JL, Chhabildas LC. Laser interferometer measurements of refractive index in shock-compressed materials. In: Gupta YM, editor. Shock Waves in Condensed Matter; 1986. p. 14.

[19] Barker LM, Hollenbach RE. Laser interferometer for measuring high velocities of any reflecting surface. J Appl Phys 1972;43:4669-75.

[20] Fowles GR. Dynamic compression of quartz. J Geophys Res 1967;72.

[21] Pope LE, Stevens AL. Wave propagation in beryllium single crystals, metallurgical effects at high strain rates, Rohde, Butcher, Holland, Karnes, eds. NY: Plenum Press, NY; 1973. p. 350-66.

[22] Grady DE. Dynamic properties of ceramic materials. Sandia National Laboratories, Report SAND94-3266, 1995.
[23] Chhabildas LC, Knudson MD. Techniques to launch projectile plates to very high velocities, high pressure shock compression of solids VII: the science and technology of high velocity impact. Berlin: Springer-Verlag; 2005.

[24] Asay JR, Chhabildas LC. Determination of the shear strength of shock compressed 6061-T6 aluminum. In: Meyers MA, Murr LE, editors. Shock waves and high-strain-rate phenomena in metals. New York: Plenum; 1981. p. $417-31$

[25] Chhabildas LC, Asay JR, Barker LM. Shear strength of tungsten under shock- and quasi-isentropic loading to $250 \mathrm{GPa}$. In. Sandia National Laboratories. 1988.

[26] Chhabildas LC, Wise JL, Asay JR. Reshock and release behavior of beryllium. In: Nellis WJ, Seaman L, Graham RA, editors. Shock Waves of Condensed Matter, $78 ; 1982$.

[27] Chhabildas LC, Asay JR. Time resolved wave profile measurements in copper to megabar pressures, In: Bachman, J., Tegner (Ed.) 8th AIRAPT Conference, pp. 183-189, 1981.

[28] Furnish MD, Chhabildas LC. Alumina strength degradation in the elastic regime. Shock Compression of Condensed Matter; 1998:501-4.

[29] Johnson Q, Mitchell AC, Smith ID. Flash x-ray tube for diffraction studies on a two-stage light-gas gun. Rev Scientific Instruments 1980;51(6):741-9.

[30] Jensen BJ, Gupta YM. X-ray diffraction measurements in shock compressed magnesium doped LiF crystals. J Appl Phys; 2006. 100, 053512 _2006. 